\title{
EVALUATION OF CURRENT DRIVE REQLIREMENTS AND \\ OPERATING CHARACTERISTICS OF A HIGH BOOTSTRAP FRACTION ADVANCED TOKAMAK REACTOR
}

\author{
W. A. Houlberg \\ S. E. Attenberger \\ Oak Ridge National Laboratory \\ P.O. Box 2009 \\ Oak Ridge, Tennessee 37831-8072
}

(615)574-1350

\section{ABSTRACT}

The reactor potential of some advanced physics operating modes proposed for the TPX physics program [1] are examined. A moderate aspect ratio $(A=4.5$ as in TPX), $2 \mathrm{GW}$ reactor (see Table I for parameters) is analyzed because of its potential for steady-state, noninductive operation with high bootstrap current fraction. Particle, energy and toroidal current equations are evolved to steady-state conditions using the $1-1 / 2-D$ time-dependent WHIST transport code [2]. The solutions are therefore consistent with particle, energy and current sources and assumed transport models. Fast wave current drive (FWCD) provides the axial seed current. The bootstrap current typically provides $80-90 \%$ of the current, while feedback on the lower hybrid current drive (LHCD) power maintains the total current. The sensitivity of the plasma power amplification factor, $Q \equiv P_{\text {fus }} / P_{\text {aux }}$, to variations in the plasma properties is examined. The auxiliary current drive power, $P_{\text {aux }}=P_{\mathrm{LH}}+P_{\mathrm{FW}}$; bootstrap current fraction; current drive efficiency; and other parameters are evaluated. The plasma is thermodynamically stable for the energy confinement model assumed (a multiple of ITER89P). The FWCD and LHCD sources provide attractive control possibilities, not only for the current profile, but also for the total fusion power since the gain on the incremental auxiliary power is typically $10-30$ in these calculations when overall $Q \approx 30$.

TABLE I. Advanced Tokamak Reactor Parameters

\begin{tabular}{llc}
\hline$R$ & Major radius & $7.28 \mathrm{~m}$ \\
$a$ & Minor radius & $1.62 \mathrm{~m}$ \\
$\kappa$ & Elongation & 2.0 \\
$\delta$ & Triangularity & 0.5 \\
$B_{t}$ & Toroidal field & $6.64 \mathrm{~T}$ \\
$I$ & Plasma current & $9.4 \mathrm{MA}$ \\
$Z_{\text {eff }}$ & C and O & 2.0 \\
$P_{\text {fus }}$ & Fusion power & $2 . \mathrm{GW}$ \\
\hline
\end{tabular}

\section{CURRENT DRIVE AND TRANSPORT MODELS}

We use a simplified analytic model for the FW power deposition profile that is proportional to the the local wave damping rate, $k_{i}[3]$, but reduced in the center of the plasma to the extent that waves are absorbed while propagating to the core [4]:

$$
h_{\mathrm{FW}}(\rho)=\frac{k_{i}(\rho) e^{-2 \int_{\rho}^{a} d s k_{i}(s)}}{\int_{0}^{a} d \rho V^{\prime}(\rho) k_{i}(\rho) e^{-2 \int_{\rho}^{a} d s k_{i}(s)}}
$$

where the normalization is such that $h_{\mathrm{FW}}$ integrated over the plasma volume is unity. The prime designates the derivative with respect to the flux surface radial coordinate $\rho$ (where $\rho^{2}$ is proportional to the toroidal flux enclosed by the surface), $k_{i}=k_{\perp}(\sqrt{\pi} / 4) \beta_{e} \xi \exp \left(-\xi^{2}\right)$, $k_{\perp}=\operatorname{Re}\left\{k_{\perp}\right\}=\left(\omega / v_{A}\right)=(\omega / c)\left(\omega_{p i} / \Omega_{i}\right), \beta_{e}=$ $n_{e} T_{e} /\left(B^{2} / 2 \mu_{0}\right), \xi=v_{\text {phase }} / v_{e}=\left(\omega / k_{\|}\right) \sqrt{m_{e} / 2 k T_{e}}$, $\omega_{p i}^{2}=4 \pi n_{i} Z_{i}^{2} e^{2} / m_{i}, \Omega_{i}=Z_{i} e B / m_{i}, Z_{i}$ is the main ion charge, $n_{i}\left(n_{e}\right)$ is the ion (electron) density, $T_{i}\left(T_{e}\right)$ is the ion (electron) temperature, $m_{i}\left(m_{e}\right)$ is the ion (electron) mass. $B$ is the magnetic field, $k_{\|}$is the parallel wave number, and $\omega$ is the wave frequency with all units in mks.

The LH power deposition profile is characterized by a gaussian width. $\Delta_{\mathrm{LH}}$, and centered at a minor radius $\rho_{\mathrm{LH}}$ :

$$
h_{\mathrm{LH}}(\rho)=\frac{e^{-\left(\rho-\rho_{\mathrm{LH}}\right)^{2} / \Delta_{\mathrm{LH}}^{2}}}{\int_{0}^{a} d \rho V^{\prime}(\rho) e^{-\left(\rho-\rho_{\mathrm{LH}}\right)^{2} / \Delta_{\mathrm{LH}}^{2}}}
$$

The FWCD calculations use a reference frequency of $81 \mathrm{MHz}$ and $k_{11}=5.43 \mathrm{~m}^{-1}$ corresponding to an electron temperature of $25 \mathrm{keV}$ at $\xi=1$. The LHCD calculations assume $N_{\|}=1.8$. These values are used in the expressions of Ehst and Karney [5] for evaluating the local current drive efficiency and currents.

*Research sponsored by the Office of Fusion Energy, U.S. Department of Energy, under contract DE-AC0584OR21400 with Martin Marietta Energy Systems, Inc. 


\section{DISCLAIMER}

Portions of this document may be illegible in electronic image products. Images are produced from the best available original document. 
The transport model for the particle, $\Gamma$, and heat fluxes. $Q$, is a combination of 'full' neoclassical [6] (i.e., including all force terms in the transport fluxes), plus a 'diagonal' anomalous contribution (i.e., $\Gamma_{j}^{\mathrm{an}} \propto \frac{\partial n_{j}}{\partial \rho}$, $\left.Q_{j}^{a n} \propto \frac{\partial T_{j}}{\partial \rho}\right):$

$$
\begin{aligned}
\left(Q_{j}, \Gamma_{j}\right) & =\left(Q_{j}, \Gamma_{j}\right)^{\text {neo }}+\left(Q_{j}, \Gamma_{j}\right)^{\text {an }} \\
Q_{j}^{\text {an }} & =-n_{j} \chi_{j}^{\text {an }} \frac{\partial T_{j}}{\partial \rho} \\
\Gamma_{j}^{\text {an }} & =-D_{j}^{\text {an }} \frac{\partial n_{j}}{\partial \rho} \\
\chi_{e}^{\text {an }} & =\chi_{i}^{\text {an }}=3 D_{j}^{\text {an }}=C^{\text {an }} g(\rho) \\
g(\rho) & =1+4(\rho / a)^{2}
\end{aligned}
$$

Transport coefficients are normalized (through $C^{\text {an }}$ ) to match a global $\tau_{E}$ expressed as the minimum of either neo-Alcator or a multiple $(H)$ of ITER L-mode confinement [7]:

$$
\begin{aligned}
\tau_{E} & =\min \left(\tau_{\mathrm{NA}}, H \times \tau_{\text {TTER89P }}\right) \\
\tau_{\mathrm{NA}_{\mathrm{A}}} & =0.07 \bar{n}_{e 20} a R^{2} q_{*} \\
q_{*} & \equiv \frac{5 a B_{t}}{I_{M}}\left(\frac{a}{R}\right) f(\kappa, \delta) \\
f(\kappa, \delta) & =\frac{\left[1+\kappa^{2}\left(1+2 \delta^{2}\right)\right]}{2} \\
\tau_{I T E R 89 P} & =0.048 \frac{A_{i}^{0.05} I_{M}^{0.85} R^{1.2} a^{0.3} \kappa^{0.5} n_{e 20}^{0.1} B_{t}^{0.2}}{P_{\mathrm{tot}}^{0.5}} \\
P_{\text {tot }}(\mathrm{MW}) & =P_{\mathrm{OH}}+P_{\alpha}+P_{\mathrm{RF}}-P_{\mathrm{rad}}
\end{aligned}
$$

where the subscript 20 on the electron density designates units of $10^{20} \mathrm{~m}^{-3}\left(\bar{n}_{20}\right.$ is the line-average and $n_{e 20}$ is the volume average), $I_{M}$ is the toroidal current in MA and $A_{i}$ is the average atomic mass number of the ions. Neoclassical electrical resistivity is used to evolve the current profile from initial conditions to steady-state. However. since the steady-state solutions are fully noninductive the resistivity model only affects the dynamics and not the steady-state solutions. The neoclassical bootstrap current model is from Hirshman [8]. The MHD equilibrium solutions are self-consistently evaluated from the evolving current and pressure profiles, although MHD stability of these profiles is not evaluated.

\section{CURRENT DRIVE REQUIREMENTS}

Figure 1 illustrates a scan of density with no inductive current. All cases were evolved for $1000 \mathrm{~s}$ to allow the current profile to approach steady-state. Feedback control on the FWCD power was used to give $q(0)<2.5$, and feedback on the LHCD maintained $I=9.4 \mathrm{MA}$. As the operating density is increased over the series of runs, the fusion power increases and $\mathrm{CD}$ requirements are reduced because of the increasing bootstrap current contribution. At an operating density of $\left\langle n_{e}\right\rangle \approx 1.75 \times 10^{20} \mathrm{~m}^{-3}, 2 \mathrm{GW}$ of fusion power is produced. The auxiliary power requirements are $P_{\mathrm{FW}}=34 \mathrm{MW}$ and $P_{\mathrm{LH}}=29 \mathrm{MW}$ at this operating point, giving a high plasma $Q(\approx 32)$ even though the CD efficiency is low $\left(\gamma=n_{e 20} I R / P_{\mathrm{RF}} \approx 0.3\right)$. Cases with other choices of $k_{\|}$show that the FWCD power may be further reduced with a corresponding increase in $Q$. In other cases it was found that reducing the $\mathrm{H}$-factor to 1.5 reduces the power gain to $Q \approx 10$ because of the lower operating temperatures and higher densities required to attain the same fusion power output.

A diagonal neoclassical model gives stronger accumulation of helium ash (and reduced performance) relative to the full neoclassical cases shown in the figures (which typically have $n_{\alpha} / n_{e} \approx 5 \%$ ). In other words, for these machine and plasma parameters, the hollowed out fuel density profile created by the fusion sink in the core and the net inward flow of fuel ions drives the helium outward. This is contrary to the common perception of neoclassical effects driving impurities inward. The effect of the full neoclassical terms is more noticeable in these calculations because there is no sawtooth activity in the core. The difference between the full and diagonal neoclassical transport models becomes even greater when suppression of the anomalous transport contribution is decreased in regions where $\nabla q<0$.

The current profile evolves to a relatively broad profile with low or negative shear over the inner $50-60 \%$ of the minor radius and $q>2$ everywhere when the FWCD maintains $2.4<q(0)<2.5$. Such low or negative shear conditions with $q(0)>0$ have been shown to yield improved core confinement in JET pellet enhanced performance (PEP) [9], Tore Supra lower hybrid enhanced performance (LHEP) [10], and DIII-D very high (VH) mode experiments [11]. In the DIII-D experiments. MHD stability evaluations show the enhanced core confinement is correlated with entry to the second stability regime [11]. Therefore, we examine the effects of current drive source on establishing low or negative shear in the core.

Figures 2 and 3 show the effect of the width and position, respectively, of the assumed $\mathrm{LH}$ power deposition on the reverse shear region. However, these calculations do not include a reduction in the transport properties in the negative shear regions. We typically find a negative shear region near the plasma center that is governed by the bootstrap current profile, which is in turn determined by the assumed radial profile of the particle and thermal diffusivities. A second negative shear region develops just inside the peak of the LH deposition. As the density is increased in all the cases shown in both Figures 2 and 3, the LH-driven negative shear region diminishes because the bootstrap current fraction is increasing and the $\mathrm{LH}$ current is decreasing. Between these two regions is a region of very low shear. Figures 2 and 3 show that when the width of the LH deposition is decreased or moved outward. the LH-driven 
ORNL-DWG 94M-3130 FED
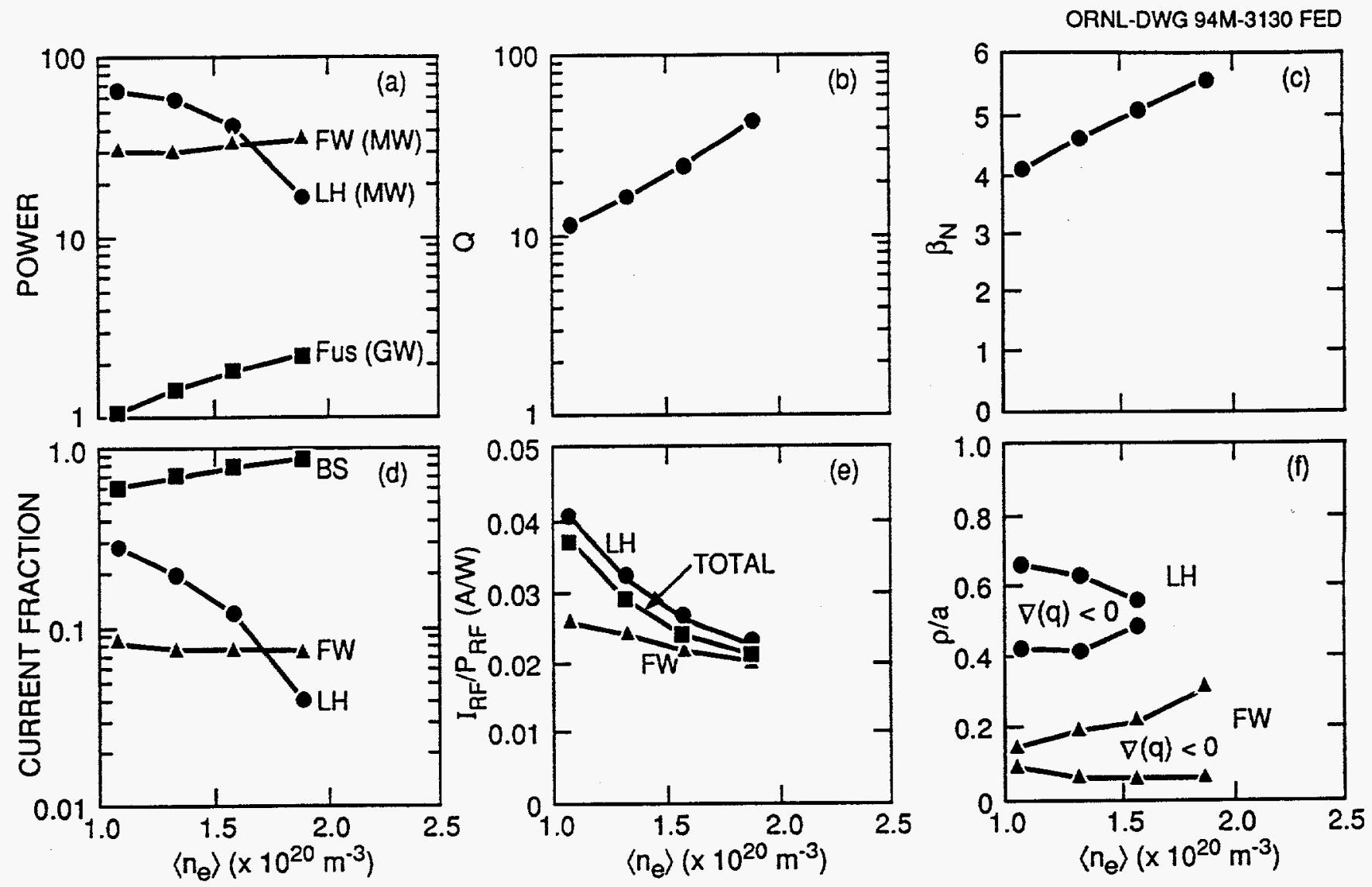

FIG. 1. Variation of plasma properties with average electron density for fully non-inductive operation with $H=2$, $\rho_{\mathrm{LH}} / a=0.6$ and $\Delta_{\mathrm{LH}} / a=0.1$. The target operating fusion power of $2 \mathrm{GW}(\mathrm{a})$ is attained at $\left\langle n_{\mathrm{e}}\right\rangle \approx 1.75 \times 10^{20} \mathrm{~m}^{-3}$, has plasma $Q \approx 32(\mathrm{~b}), \beta_{N} \approx 5.3(\mathrm{c}), 85 \%$ bootstrap current fraction (d), overall current drive efficiency $\approx 0.025 \mathrm{~A} / \mathrm{W}$ (e), and low or negative shear over the region $r / a \leq 0.6$ (f).
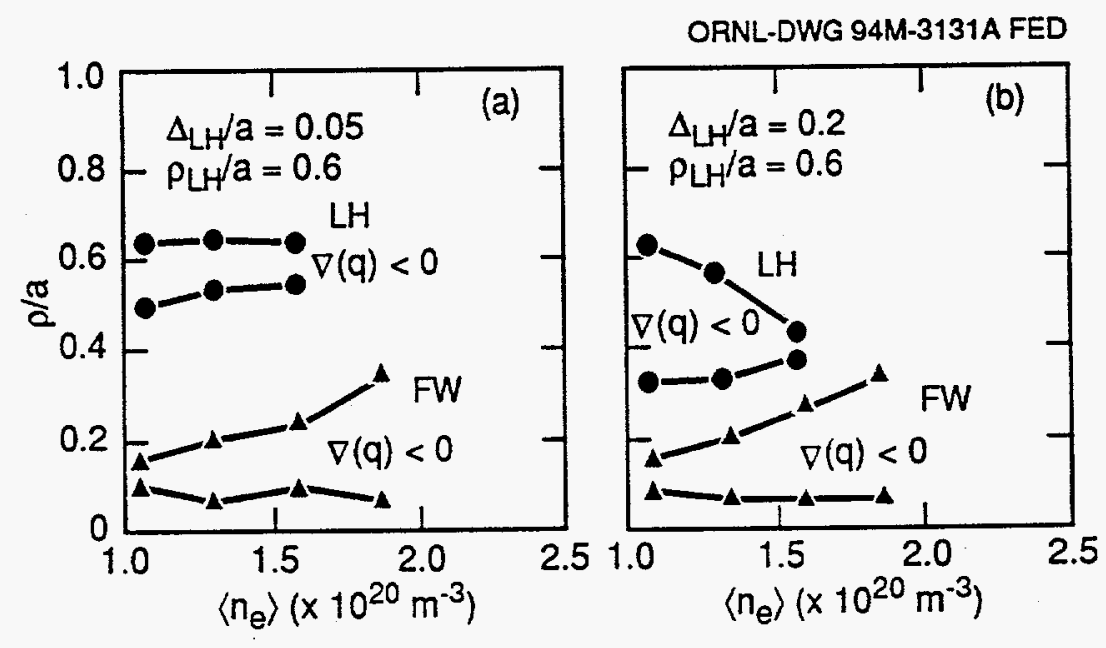

FIG. 2. Evolution with increasing density of the negative shear regions for narrower, $\Delta_{\mathrm{LH}} / a=0.05$ (a). and broader, $\Delta_{\mathrm{LH}} / a=0.2$ (b). LH power deposition profiles. 

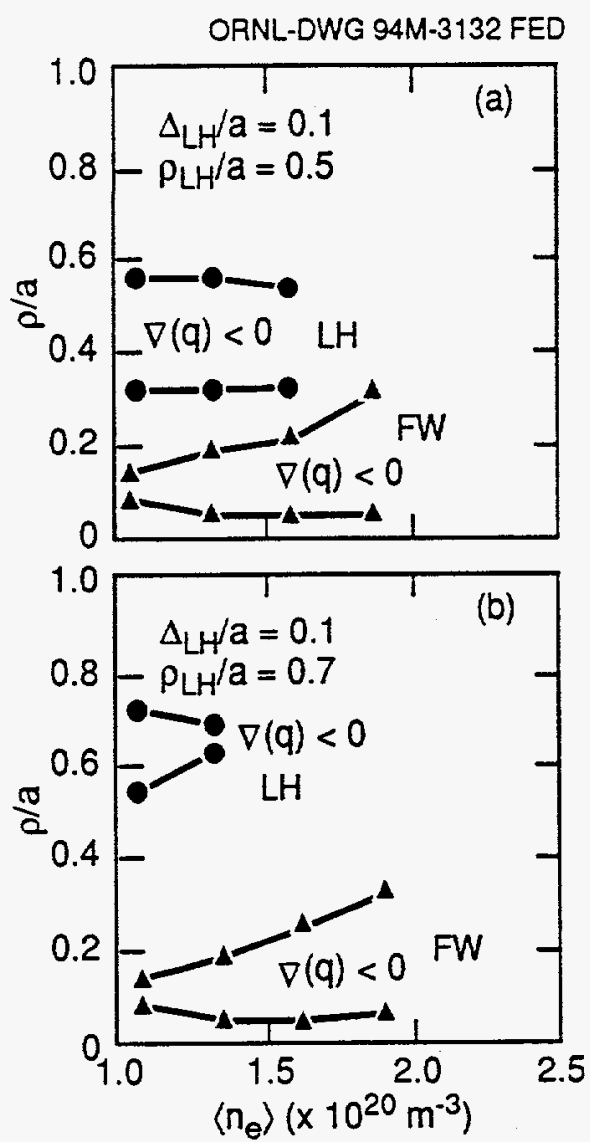

FIG. 3. Evolution with increasing density of the negative shear regions for deeper, $\rho_{\mathrm{LH}} / a=0.5(\mathrm{a})$, and shallower, $\rho_{\mathrm{LH}} / a=0.7$ (b), LH power deposition profiles.

negative shear region is narrower. Moving it inward and broadening it makes it wider and nearly merge with the bootstrap-driven negative shear region.

In other cases, a narrower $\mathrm{FW}$ deposition profile was found to reduce the total FW current because only $J(0)$ is critical for the FW source. Then the LHCD requirements are increased and more control over the negative shear regions is obtained. In some cases the negative shear regions merged and covered $60 \%$ of the minor radius. These calculations suggest that control over the position from $\rho / a \approx 0.5-0.6$ and deposition width of the outer CD source should have a strong effect on shear, and therefore, on the confinement properties. But, as mentioned earlier, the radial profiles of the transport coefficients also play a significant role in the $q$ profile through their determination of the density and temperature profiles and the bootstrap current.

In cases where the anomalous transport coetficients were reduced in negative shear regions, several interesting observations have been made. First of all. the thermal transport of electrons and ions have significant contributions in the core from synchrotron radiation and neoclassical conductivity, respectively. Reducing the anomalous transport coefficients by a factor of 35 in the core therefore, did not significantly increase energy confinement. Reducing the particle diffusivity made the importance of the full neoclassical terms even more apparent. with alpha ash accumulation being a severe problem with a diagonal neoclassical model. The full neoclassical terms helped reduce the accumulation to manageable levels. Secondly, the negative shear regions are modified by the suppression of transport coefficients primarily because they allow stronger temperature gradients to develop just inside the region of suppressed transport. When the outer, LH, current drive region is too far out $\left(\rho_{\mathrm{LH}} / a \approx 0.7\right)$ or too weak, the increased bootstrap contribution can allow the negative shear region to collapse. This has also been found in simulations of other high bootstrap fraction tokamak reactors $[12,13]$.

\section{THERMODYNAMIC STABILITY AND CONTROL}

Because of the dependence of plasma confinement properties on total current as well as the dependence of bootstrap current on confinement properties through the kinetic pressure profile, plasmas operating with high bootstrap current fraction may be susceptible to either decay or runaway growth instead of simply exhibiting high gain factors (for the current and power). That is, if the plasma energy content drops, the bootstrap current driven by the pressure decreases and the total plasma current decreases. When confinement and current are positively correlated, the plasma confinement decreases further, reinforcing the original excursion. Whether it results in a either a high gain or an unstable situation is governed by the plasma resistivity and total inductance as well as the relationship between plasma current and confinement. We have assessed the thermodynamic stability issue with time dependent calculations in which the LH and FW powers and the transformer currents are fixed. The plasma is then perturbed from equilibrium and its evolution is followed for several inductive $(L / R)$ times. All cases examined have been thermodynamically stable even though the confinement and current are strongly linked. Figure 4 shows a set of profiles after $2000 \mathrm{~s}$ simulation of a $2 \mathrm{GW}$ case using the parameters of Fig. 1.

Control of the steady-state operating conditions has been examined by perturbing the LH and FW sources and examining the incremental power amplification factor (e.g., $Q_{\Delta \mathrm{LH}} \equiv \Delta P_{\text {fus }} / \Delta P_{\mathrm{LH}}$ ), the change in axial safety factor. and the change in plasma operating current. Even with low $C D$ fractions the current drive sources provide strong leverage for plasma control. The axial safety factor is proportional to $P_{\mathrm{FW}}$ when the power deposition is peaked on axis, allowing $q(0)$ to be controlled (albeit on a long timescale) by the FW source. The LH system provides leverage on both the total power output and total plasma current at constant 

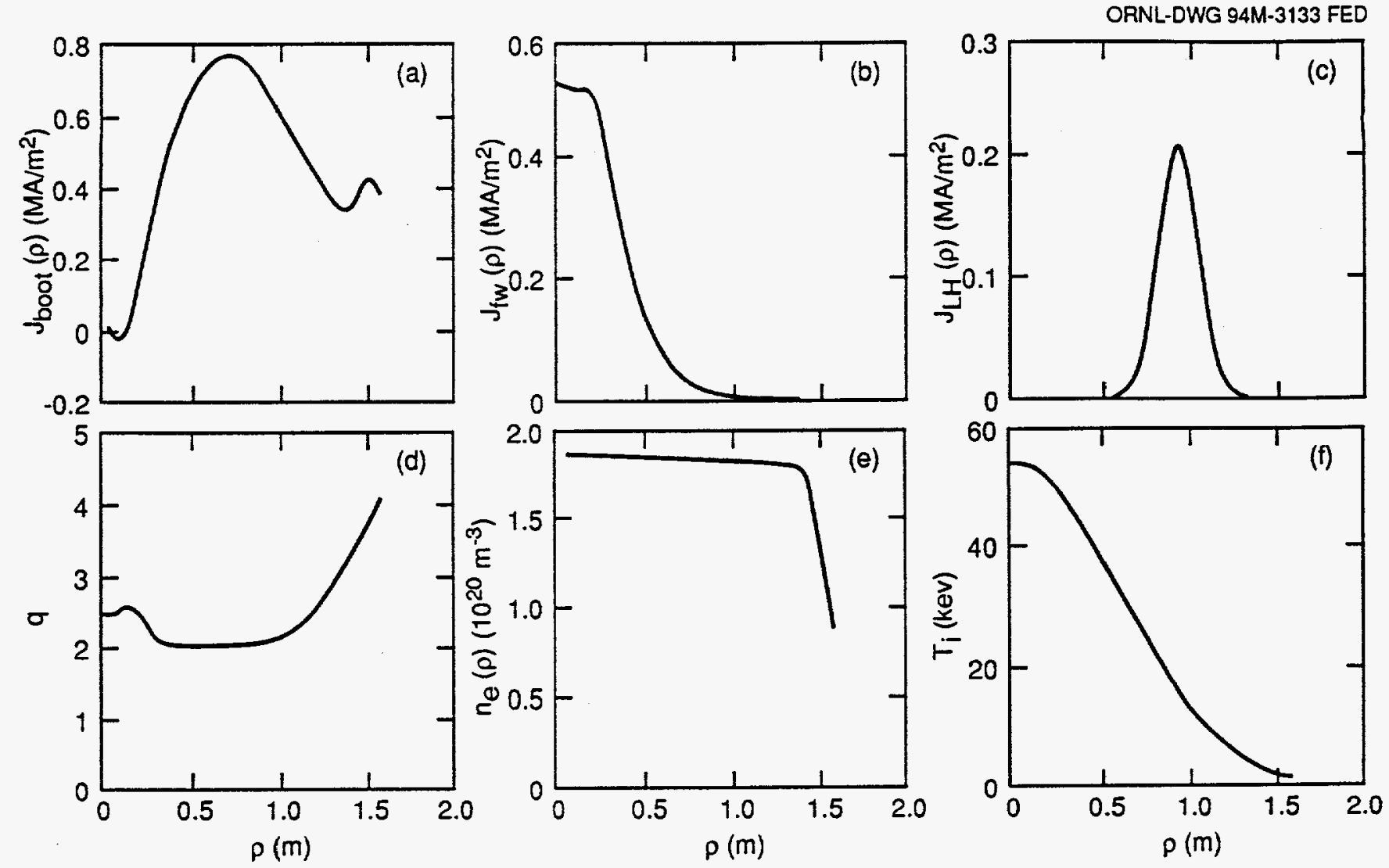

FIG. 4. Steady-state radial profiles of the bootstrap current density (a), FW current density (b), LH current density (c), safety factor (d), electron density (e) and ion temperature (f), for $P_{\mathrm{FW}}=33.9 \mathrm{MW}, P_{\mathrm{LH}}=29.3 \mathrm{MW}, P_{f u s}=2.13 \mathrm{GW}$, $I=9.59 \mathrm{MA}$.

operating density. When we reduce the LH power for the case shown in Fig. 4 to $23 \mathrm{MW}$ (from $29.3 \mathrm{MW}$ ), the LHCD decreases by $160 \mathrm{kA}$ while the total current decreases by $500 \mathrm{kA}$ (a gain factor of 3 ). The fusion power decreases by $150 \mathrm{MW}$ (a gain factor of 25 on the power delivered to the plasma). As can be seen in Fig. 4, the current density is relatively high near the plasma edge from the bootstrap current driven by the steep density and temperature gradients. The plasma boundary conditions (i.e., divertor operating conditions. impurity levels, etc.) are expected to have a strong influence on these profiles. Although it is an important element of the advanced tokamak program, it is beyond the scope of the present study.

\section{SUMMARY}

We have addressed several aspects of fully noninductive current drive in an advanced tokamak of moderate aspect ratio. Plasma simulations have shown several interesting and attractive features including: the desirability of a least two current sources, one for axial seed current density (e.g., FWCD) and one for off-axis control of the total current (e.g., LHCD); tunable position and width of the outer source for control of the safety factor and shear profiles; thermodynamic stability of the solutions; high gain factors on total fusion power and current as the CD sources are varied at constant plasma density; the importance of utilizing the full complement of neoclassical terms for the particle transport since it governs helium ash accumulation; and the importance of synchrotron radiation transport in the core. Much more evaluation of the physics issues still needs to be performed as part of the advanced tokamak program. These include more detailed MHD stability evaluations, sensitivity to variations in transport models (including both parametric dependence and radial form), correlations between MHD stability and transport, details of the RF systems and physics models, and overall system efficiencies.

\section{ACKNOWLEDGEMENTS}

This research was sponsored by the Office of Fusion Energy, U.S. Department of Energy, under contract DEAC05-840R21400 with Martin Marietta Energy Systems, Inc. 


\section{REFERENCES}

[1] G.H. Neilson, et al., "Mission and Physics Design of the Tokamak Physics Experiment," this proceedings.

[2] W.A. Houlberg, S.E. Attenberger, L.M. Hively, Nucl. Fusion 32 (1982) 935

[3] M. Porkolab, in Radio Frequency Power in Plasmas 1992 (Proc. 9th Topical Conf. Charleston, SC, 1991), American Institute of Physics, New York (1992) 197.

[4] D.B. Batchelor, private communication, April 1994.

[5] D.A. Ehst, C.F.F. Karney, Nucl. Fusion 31 (1991) 1933.

6] F.L. Hinton, R.D. Hazeltine, Rev. Modern Phys. 48 (1976) 239.

[7] N.A. Uckan, P.N. Yushmanov, T. Takizuka, et al., in Plasma Physics and Controlled Nuclear Fusion Re- search 1990 (Proc. 13th Int. Conf. Washington, DC, 1990), Vol. 3, IAEA, Vienna (1991) 307.

[8] S.P. Hirshman, Phys. Fluids 31 (1988) 3150.

[9] M. Hugon, B.Ph. van Milligan, P. Smeulders, L.C. Appel, D.V. Bartlett, et al., Nucl. Fusion 32 (1992) 33.

[10] D. Moreau, B. Saoutic, G. Agarici, B. Beaumont, A. Becoulet, et al., in Plasma Physics and Controlled Nuclear Fusion Research 1992 (Proc. 14th Int. Conf. Würzburg, 1992), Vol. 1, IAEA, Vienna (1993) 649.

[11] E.A. Lazarus, et al., "The Role of Shaping in Achieving High Performance in DIII-D," in Plasma Physics and Controlled Nuclear Fusion Research 1994 (Proc. 15th Int. Conf. Seville, 1994), to be published.

[12] H. St. John, Dallas TTF Meeting, April 1994.

[13] D. Boucher. Dallas TTF Meeting, April 1994.

\section{DISCLAIMER}

This report was prepared as an account of work sponsored by an agency of the United States Government. Neither the United States Government nor any agency thereof, nor any of their employees, makes any warranty, express or implied, or assumes any legal liability or responsibility for the accuracy, completeness, or usefulness of any information, apparatus, product, or process disclosed, or represents that its use would not infringe privately owned rights. Reference herein to any specific commercial product, process, or service by trade name, trademark, manufacturer, or otherwise does not necessarily constitute or imply its endorsement, recommendation, or favoring by the United States Government or any agency thereof. The views and opinions of authors expressed herein do not necessarily state or reflect those of the United States Government or any agency thereof. 